\title{
Clinical Trial Principal Investigator Curriculum Vitae
}

National Cancer Institute

\section{Source}

National Cancer Institute. Clinical Trial Principal Investigator Curriculum Vitae. NCI

Thesaurus. Code C115487.

Records detailing the education and work experience of the principal investigator of a clinical trial. 